\title{
Employer Challenges With the Chronic Kidney Disease Population
}

\author{
Sean Sullivan, JD
}

\begin{abstract}
BACKGROUND: Employers have not yet begun to focus on chronic kidney disease (CKD) as a major health issue in the workplace. CKD is part of the metabolic and cardiovascular health burden, which is a major threat to the future survivability of the employer-based health financing system. It represents one opportunity for employers to intervene now to save health care costs later.
\end{abstract}

OBJECTIVE: To describe the impact of CKD in non-Medicare payer populations (i.e., employer group health plans) and the importance of early screening and detection of the disorder to improve patient quality of life and reduce the future cost burden of this disease.

SUMMARY: Twenty million Americans suffer from CKD, and 20 million more are at elevated risk; soon, 1 in 9 Americans will have CKD. Control of comorbidities may slow its progression, and 2 are critical-type 2 diabetes and hypertension. Health care costs are made up of 2 categories and 5 subcategories: direct costs, which are (1) inpatient, (2) outpatient, and (3) drug costs-roughly three quarters of the total costs associated with patients who have nondialysis renal insufficiency —and indirect costs, which are lost productivity due to (4) disability and (5) absenteeism.

CONCLUSION: CKD should be considered part of a larger picture of metabolic health. As CKD is related to cardiometabolic health, it is beginning to capture payers' and purchasers' interest. Investing health care dollars today to identify the disease and slow its progression should improve patient quality of life and reduce the future cost burden of this disease and its associated conditions.

J Manag Care Pharm. 2007:13(9):S19-S21

Copyright $\odot$ 2007, Academy of Managed Care Pharmacy. All rights reserved.

\section{Author}

SEAN SULLIVAN, JD, is cofounder, president, and chief executive officer, Institute for Health and Productivity Management, and chief executive officer, Initiative for Value-Based Health Benefits. He is also editor-in-chief of the quarterly magazine Health \& Productivity Management.

AUTHOR CORRESPONDENCE: Sean Sullivan, JD, President \& CEO, Institute for Health and Productivity Management, Gainey Ranch Center, 7702 E. Doubletree Ranch Rd., Suite 300, Scottsdale, AZ 85258. Tel.: 480.607.2660.E-mail: sean@ihpm.org
E mployers have historically focused on health care issues with immediate cost implications, such as mental health or diabetes. However, for close to a decade, the Institute for Health and Productivity Management (IHPM) has been working with employers to improve their employees' health and to maximize the employers' return on their investment in workers health. Increasingly, this evidence-based work is attracting attention. Good, solid information on total costs, including lost productivity, supports a change in how employers will view and pay for health care—as a targeted "investment" in better health and as a business asset, rather than just a "cost" of doing business.

\section{The Long-Term Perspective: CKD}

Chronic kidney disease (CKD) is an important and expensive workplace issue that has typically been overlooked by employers in favor of issues that affect a greater proportion of the population, such as metabolic health and mental health. IHPM held a series of employer advisory boards on CKD last year, structured to help employer medical directors consider how CKD fits into a larger picture of metabolic disease and cardiovascular health. IHPM research and study results demonstrate that metabolic and cardiovascular risk factors and conditions create a significant cost burden for employers and a potential threat to the future of employer-based health financing. CKD is part of the overall health care cost burden, and represents one opportunity for employer investment and intervention with the goal of reducing future health care costs. Both productivity and medical costs contribute to the staggering implications of inaction. Four facts highlight the need for employer-based health care plans to begin addressing CKD more aggressively:

1 Twenty million Americans suffer from CKD, while 20 million more are at elevated risk. ${ }^{1}$

2. Nearly half of patients with CKD are unaware they have it. ${ }^{1}$ Without aggressive management, kidney dysfunction progresses to end-stage renal disease (ESRD), with subsequent dialysis or transplant needs. ${ }^{1}$

3. Control of CKD comorbidities may slow CKD's progression. Control of type 2 diabetes and hypertension is known to slow CKD progression, while efforts to address other areas of cardiometabolic health are also considered critical. ${ }^{2}$

4. Anemia treatment in CKD is associated with improved outcomes and decreased health care costs. ${ }^{3}$

CKD is a costly disease for every payer type. Treatment costs roughly double as CKD progresses from one stage to the next. Data from the IHPM database (2001) indicate that insurance claims range from $\$ 5,000$ to $\$ 12,000$ per patient per year as a patient proceeds from stage 1 to stage 2 . At stages 3 and 4 , claims increase to $\$ 15,000$ to $\$ 28,000$ per year, partly due to serious associated comorbidities such as heart failure and atherosclerosis. At stage 5, ESRD, costs can exceed $\$ 70,000$ per patient per year. ${ }^{3,4}$ It is reasonable to assume these costs will 
increase if nothing is done to halt the progression of CKD, making it a financially important issue for employers.

\section{CKD's Workplace Costs}

Health care costs can be sorted into 2 main categories and 5 subcategories. The first main category is direct costs, which are (1) inpatient, (2) outpatient, and (3) drug costs—roughly three quarters of the total costs associated with patients who have nondialysis renal insufficiency. The second main category is indirect costs, which are lost productivity due to (4) disability and (5) absenteeism. CKD has direct and indirect costs that ripple into the workplace. Employees with CKD may be eligible for disability, while others may frequently be absent due to illness or medical appointments. Generally, disability and absenteeism account for about $25 \%$ of the total health-related costs of CKD. ${ }^{4}$ As many employers no longer divide workplace leave into sick days and other types of leave, this estimate may be low.

A 6th type of cost is worthy of discussion. "Presenteeism" is the performance lost daily in the workplace when employees come to work in spite of illness, which has negative repercussions on business performance. Researchers have established presenteeism measurement databases for conditions including depression, seasonal allergies, and migraine. Although presenteeism has not yet been measured for CKD, researchers have been able to measure presenteeism in kidney disease. ${ }^{3}$

\section{Collaborating to Fully Understand the Economic Impact of Anemia}

Anemia is a serious concern and of considerable cost in CKD. Although much work has been done concerning anemia resulting from chemotherapy treatment of cancer patients, little work has looked at the economic impact of untreated anemia in nondialysis CKD patients. A Nephrology Outcomes/Collaborative Research study examined the health and disability claims of 600,000 people over 3.5 years (January 1998 to June 2001) (Table). Seven Fortune 500 companies provided information for the combined database. The researchers determined that of 992 CKD patients who were not receiving anemia therapy, 176 had an ICD-9-CM (International Classification of Diseases, Ninth Revision, Clinical Modification) coded diagnosis of anemia. Using a multivariate regression analysis, they analyzed the costs specific to anemia for cardiovascular disease, diabetes, liver cirrhosis, transplantation, hypertension, chemotherapy, and Charles Comorbidity Index, as well as age, sex, and health plan region. They further examined the anemia data using univariate and multivariate analyses on the untreated anemia population to look at the associated direct and indirect costs. ${ }^{5}$

The statistically significant findings from the univariate model in monthly cost differences indicate that the $176 \mathrm{CKD}$ patients who were not treated for anemia incurred 2.5 more direct costs and 1.4 more indirect costs than did the CKD patients who did not have an ICD-9-CM code for anemia. ${ }^{5}$ The

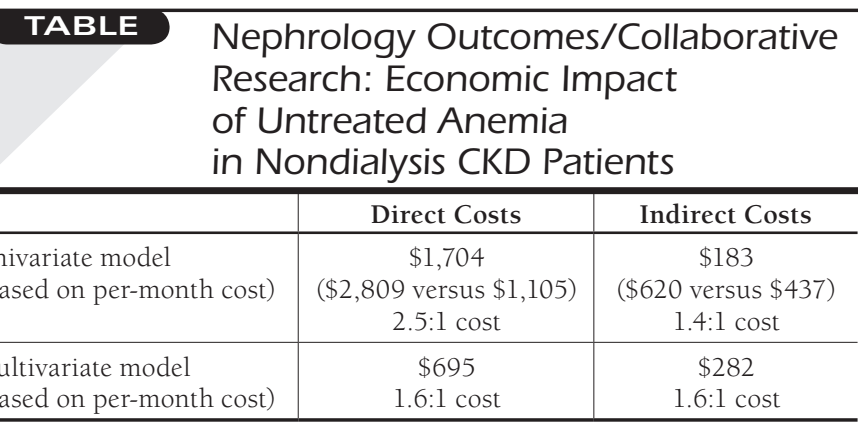

Adapted from Mody SH (2004). ${ }^{6}$

$C K D=$ chronic kidney disease

multivariate model, which takes into account comorbidities and other factors, indicates that for nondialysis CKD patients, the indirect cost ratio was approximately 1.6:1. The direct cost difference was also 1.6:1.

Recognizing that predicted increases in ESRD incidence, prevalence, and cost between now and 2010 will be significant and that slowing ESRD's progression could reduce costs, Trivedi et al. developed a mathematical model to assess the economic impact of decreasing chronic renal failure (CRF) progression by $10 \%, 20 \%$, and $30 \%{ }^{7}$ The model indicates that without intervention, the average decline in glomerular filtration rate (GFR) in subjects with CRF is approximately $7.56 \mathrm{~mL}$ per minute per year. The model calculates the time gained before onset of ESRD for different degrees of reduction in the progression of CRF. Slowing GFR decline by $10 \%, 20 \%$, or $30 \%$ over 10 years in patients with GFRs of $60 \mathrm{~mL}$ per minute or less was calculated to result in reduced medical care spending of $\$ 18.5$ billion, $\$ 39$ billion, and $\$ 60$ billion, respectively. In patients with GFRs of $30 \mathrm{~mL}$ per minute or less, a 10\%, 20\%, and 30\% decrease in the rate of decline would result in estimated cumulative savings over 10 years equal to $\$ 9.06, \$ 19.98$, and $\$ 33.37$ billion, respectively. Of interest were the patientperspective findings. Approximately four fifths of subjects with CRF $(n=113)$ considered a few weeks of being dialysis free to be of great value $(P \leq 0.001)$, which could be achieved with a $10 \%$ reduction in the rate of decline in GFR. ${ }^{7}$ In addition, as determined by a written questionnaire, patients expressed a willingness to follow a restricted diet, take 6 extra medications per day, and make 6 extra office visits per year to slow the progression of renal failure. This mathematical model suggests that intensive renal-protective efforts beginning before or at stage 3 CKD could potentially result in a large cost savings. ${ }^{8}$

Most government costs will be accrued in the population of CKD patients who are no longer working, as many of these patients will qualify for Medicare services. Because employers pay a large share of the cost of Medicare through taxes, the relevance of CKD costs to employer health plans is partly 
unaccounted for. The costlier the Medicare population becomes, the more employers may have to contribute through employer Social Security taxes to fund Medicare. Employers' contribution to Medicare generally is not calculated as part of their total health care costs, but an economist's definition of total costs certainly would include it.

The president's budget for this year proposes shifting some of Medicare's future CKD-associated costs to private payers and specifically to employers by extending the time before patients would be Medicare eligible. ${ }^{8}$ The proposal would increase the time from 30 to 60 months before Medicare becomes the secondary payer for employers who cover retiree health costs for ESRD patients. In 2007 alone, projected savings for the government are $\$ 50$ million. Between 2007 and 2011, the projected savings increase to $\$ 470$ million. Shifting costs of this magnitude to employers who provide coverage for these employees and for retirees should heighten their awareness of failing to acknowledge this problem and intervene more aggressively.

\section{The CKD-Cardiometabolic Link}

CKD is frequently related to overall cardiometabolic health, which is beginning to capture payers' and purchasers' interest. Although the term "metabolic syndrome" is being debated and refined, most stakeholders agree that a related set of risk factors-cardiovascular and metabolic (mostly related to diabetes), with obesity a key component—collectively determine cardiometabolic health. Three quarters of diabetics die from heart disease or stroke, and the key factor may be atherosclerosis.

Many employer health plans and their medical leaders have little awareness of CKD's prevalence and total cost impact in the workplace. As a result of IHPM's experience with plans and employers, IHPM recommends that plans and employers consider CKD as an endpoint of diabetes and hypertension and integrate various cardiometabolic health/disease management efforts. Focusing attention on optimizing drug therapies that treat CKD comorbidities and slow its progression should prove to be one avenue of improving overall care of employees with CKD.

\section{Conclusion}

Globally, health care policy makers and researchers are beginning to place CKD in the larger framework of cardiovascular and metabolic health. Health care costs are a business issue and not just a medical issue. As shown in the Trivedi et al. ${ }^{7}$ model assessing the economic impact of decreasing CRF progression, slowing the progression of CKD will result in estimated cumulative savings over 10 years of $\$ 9.06$ to $\$ 33.37$ billion. Ultimately, slowing disease progression should lead to improved patient quality of life while reducing the cost burden of this disease and its associated conditions.

\section{DISCLOSURES}

Author Sean Sullivan discloses no potential bias or conflict of interest relating to this article.

\section{REFERENCES}

1. National Kidney Foundation. K/DOQI clinical practice guidelines for chronic kidney disease: evaluation, classification, and stratification. Am J Kidney Dis. 2002;39(2 suppl 1):S50

2. Kausz AT, Khan SS, Abichandani R, et al. Management of patients with chronic renal insufficiency in the Northeastern United States. J Am Soc Nephrol. 2001;12:1501-07.

3. McClellan W, Aronoff SL, Bolton WK, et al. The prevalence of anemia in patients with chronic kidney disease. Curr Med Res Opin. 2004;20:1501-10.

4. Boyce SP, Buteau S, Cremieux PY, Mody SH, Piech CT. Value in Health. 2003; 6(3):286 (Abstract PRK4)

5. Mody SH. Reducing the economic and clinical burden of CKD in the managed care setting. Biotech Healthcare. 2004;56-61.

6. Mody SH. Economic impact of untreated anemia in nondialysis chronic kidney disease patients: an employer's perspective. J Am Soc Nephrol. 2003;14:457A. (Abstract SA-PO727).

7.Trivedi HS, Pang MM, Campbell A, Saab P. Slowing the progression of chronic renal failure: economic benefits and patients' perspectives. Am J Kidney Dis. 2002;39:721-29.

8. Geisel J. Budget shifts more costs of renal disease to employers. Business Insurance. 2006;36: February 13. Available at: http://findarticles.com/p/articles/ mi_hb5252/is_200602. 